NASA-TM-111631

Reprinted from MONTHLy WEATHER Review, Vol. 123, No. 6, June 1995 American Meteorological Society

\title{
Vorticity and Vertical Motions Diagnosed from Satellite Deep-Layer Temperatures
}

Roy W. Spencer, William M. Lapenta, and Franklin R. Robertson 


\title{
Vorticity and Vertical Motions Diagnosed from Satellite Deep-Layer Temperatures
}

\author{
Roy W. Spencer, William M. Lapenta, and Franklin R. Robertson \\ Earth System Science Division, NASA Marshall Space Flight Center, Huntsville, Alabama
}

(Manuscript received 25 May 1994, in final form 21 November 1994)

\begin{abstract}
Spatial fields of satellite-measured deep-layer temperatures are examined in the context of quasigeostrophic theory. It is found that midtropospheric geostrophic vorticity and quasigeostrophic vertical motions can be diagnosed from microwave temperature measurements of only two deep layers. The lower- $(1000-400 \mathrm{hPa})$ and upper- $(400-50 \mathrm{hPa})$ layer temperatures are estimated from limb-corrected TIROS-N Microwave Sounding Units (MSU) channel 2 and 3 data, spatial fields of which can be used to estimate the midtropospheric thermal wind and geostrophic vorticity fields. Together with Trenberth's simplification of the quasigeostrophic omega equation, these two quantities can be then used to estimate the geostrophic vorticity advection by the thermal wind, which is related to the quasigeostrophic vertical velocity in the midtroposphere.

Critical to the technique is the observation that geostrophic vorticity fields calculated from the channel 3 temperature features are very similar to those calculated from traditional, "bottom-up" integrated height fields from radiosonde data. This suggests a lack of cyclone-scale height features near the top of the channel 3 weighting function, making the channel 3 cyclone-scale "thickness" features approximately the same as height features near the bottom of the weighting function. Thus, the MSU data provide observational validation of the LID (level of insignificant dynamics) assumption of Hirshberg and Fritsch.
\end{abstract}

\section{Introduction and background}

a. Satellite temperature retrievals versus deep-layer temperatures

The coarse vertical resolution provided by satellite temperature sounders flown to date has been regarded as seriously degrading their utility for analysis of baroclinic weather systems. Individual channels of these infrared or microwave sounders have sensitivity to deeplayer weighted average temperature, the weights being given by the channels' respective weighting functions. While the infrared sounders have had somewhat better vertical resolution than microwave sounders in the troposphere, their weighting functions are temperature profile-dependent and are more sensitive to cloud contamination.

If multiple sounder channels with sufficiently low radiometric noise have overlapping weighting functions, then they can be deconvolved into shallower "averaging kernels" (Conrath 1972; Huang et al. 1992). These averaging kernels represent the highest vertical resolution that a satellite temperature sounder can measure directly. The extent to which the vertical resolution can thereby be improved is limited by the number, vertical placement, and shape of the weighting functions, as well as the radiometric noise of the mea-

Corresponding author address: Dr. Roy W. Spencer, ES43, NASA/MSFC, Huntsville, AL 35812 surements and the accuracy of the radiative transfer theory that describes the weighting functions. To achieve still greater vertical resolution, retrieval procedures must be employed that add ancillary information, usually in the form of a climatological or modelbased first-guess temperature profile.

In order to demonstrate the utility of deep-layer temperatures for monitoring and diagnosing extratropical baroclinic weather systems, we will depart from this tradition of attempting to retrieve high vertical resolution. While deep-layer temperatures have been used for climate-monitoring purposes (e.g., Spencer and Christy 1992a,b), there has been little emphasis on the use of satellite deep-layer temperatures for directly computing baroclinic structure within individual transient events. The closest example is the assimilation of satellite radiances into numerical weather prediction (NWP) models (e.g., Eyre and Lorenc 1989) to constrain the model temperature fields, but the full vertical resolution of the model is still retained for model calculations.

Here we will develop a conceptual framework for understanding just what the satellite measurements in their raw form represent in the context of quasigeostrophic theory. In particular, we will demonstrate the utility of deep-layer temperatures for analyzing baroclinic processes in extratropical cyclones.

\section{b. Midtropospheric geostrophic vorticity and vertical motions}

A particularly difficult parameter to diagnose, even from conventional observations, is the vertical velocity. 
It is, of course, critically related to weather since vertical displacements of air parcels control precipitation, water vapor, and cloud processes and their associated latent heat and radiant energy exchanges. Durran and Snellman (1987) provided a review of the problems and merits associated with several techniques for diagnosing quasigeostrophic vertical motion from conventional data in an operational environment. They found the omega equation simplification of Trenberth (1978), which involves a single forcing term, provided excellent results that are competitive with the $Q$-vector approach (Hoskins et al. 1978). Trenberth's formulation for this simplification, which was originally addressed by Sutcliffe (1947), is

$$
\begin{aligned}
& \left(\sigma \nabla^{2}+f_{0}^{2} \frac{\partial^{2}}{\partial p^{2}}\right) \omega \\
& \quad=2 f_{0} \frac{\partial \mathbf{V}_{g}}{\partial p} \cdot \nabla\left(\frac{1}{f_{0}} \nabla^{2} \Phi\right)+\text { deformation terms, }
\end{aligned}
$$

advection of vorticity

by the thermal wind

where the notation follows Holton (1979). Trenberth notes, and Durran and Snellman (1987) agree, that midtropospheric $\omega$ can be estimated by simply the advection of geostrophic vorticity by the thermal wind. This is because the deformation terms are usually small and the $\omega$ field is typically dominated by a single sinusoidal mode, in which case the lhs term is proportional to $-\omega$. This avoids inversion of the elliptical operator on the lhs, which can lead to more computational noise than signal enhancement. In operational procedures, the vertical motion can therefore be qualitatively estimated by superimposing a midtropospheric thickness pattern on a midtropospheric vorticity pattern, two fields familiar to every forecaster. Solenoids formed by these two contoured fields would be proportional to the quasigeostrophic forcing of vertical motion.

We will demonstrate that two deep-layer temperatures are sufficient to quantitatively diagnose the geostrophic vorticity advection by the thermal wind, and thus $\omega$, in the midtroposphere. The technique builds upon both the traditional "bottom-up" quasigeostrophic perspective of height changes (e.g., Holton 1979), as well as the alternative "top down" viewpoint of Hirshberg and Fritsch (1991a,b; hereafter HF91) and Hirshberg and Fritsch (1993; hereafter HF93). HF91 and HF93 argue that height changes of a pressure surface in association with local warming can occur below the warming if a level of insignificant dynamics (LID) can be assumed in the stratosphere (near $50 \mathrm{hPa}$ ). Discussion of the LID concept and its relevance to interpretation of synoptic-scale structure in the MSU data are presented in section 4 . We will show that cyclone-scale features in MSU channel 3
(MSU3) data provide observational support for the LID assumption.

\section{Method}

\section{a. Microwave layer temperatures}

Microwave temperature sounder weighting functions, such as those from the MSU (Fig. 1), are nearly insensitive to temperature variations, so that they can be considered to be a constant function of pressure altitude. This makes their interpretation more straightforward than infrared sounders' weighting functions that vary in pressure altitude as the air temperature changes. The weighting function shapes in Fig. 1 suggest that most of the sensed radiation comes from a layer bounded by pressure surfaces, although it is obvious that these pressure surfaces are not well defined. Nevertheless, the fact that the pressure layer sensed by a microwave temperature sounder channel remains virtually constant allows us to describe MSU temperature variations in pressure coordinate system terms, such as geopotential height and thickness.

\section{b. MSU data and limb corrections}

The MSU provides eleven individual measurements (footprints) across a swath of approximately $2200-\mathrm{km}$ width. Each footprint has a spatial resolution of about $110 \mathrm{~km}$ at nadir, increasing to about $200 \mathrm{~km}$ at the end (first and eleventh) footprint positions (Fig. 2). The

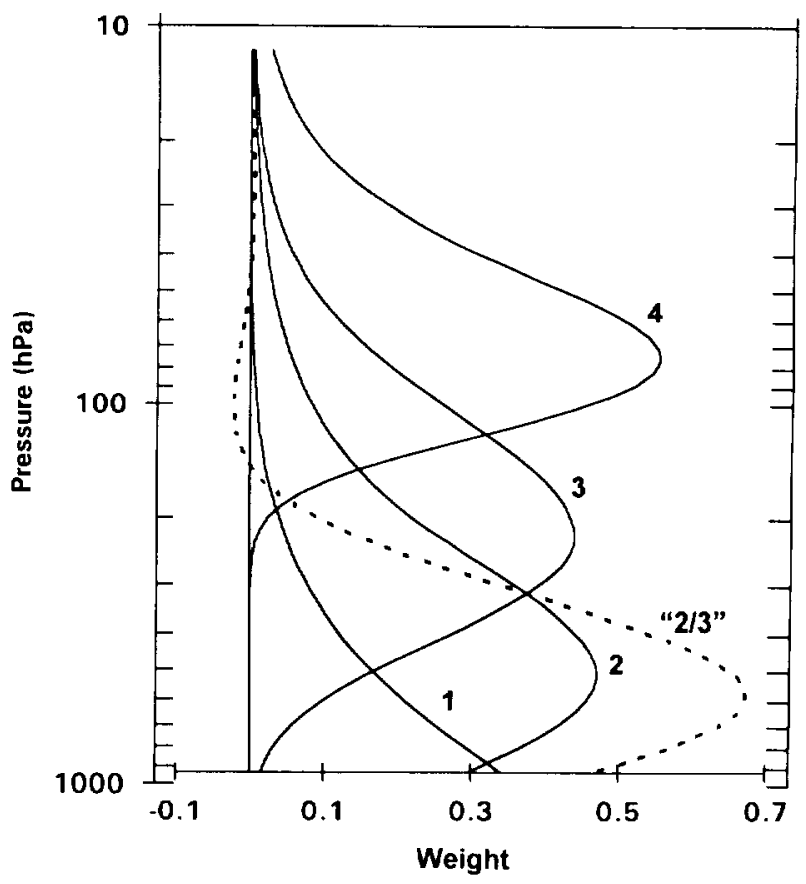

FIG. 1. Vertical profile of weighting functions for MSU channels $1-4$, and an averaging kernel for synthesized channel " $2 / 3$." The weight equals the change of transmittance with $\log _{e}(p)$. 


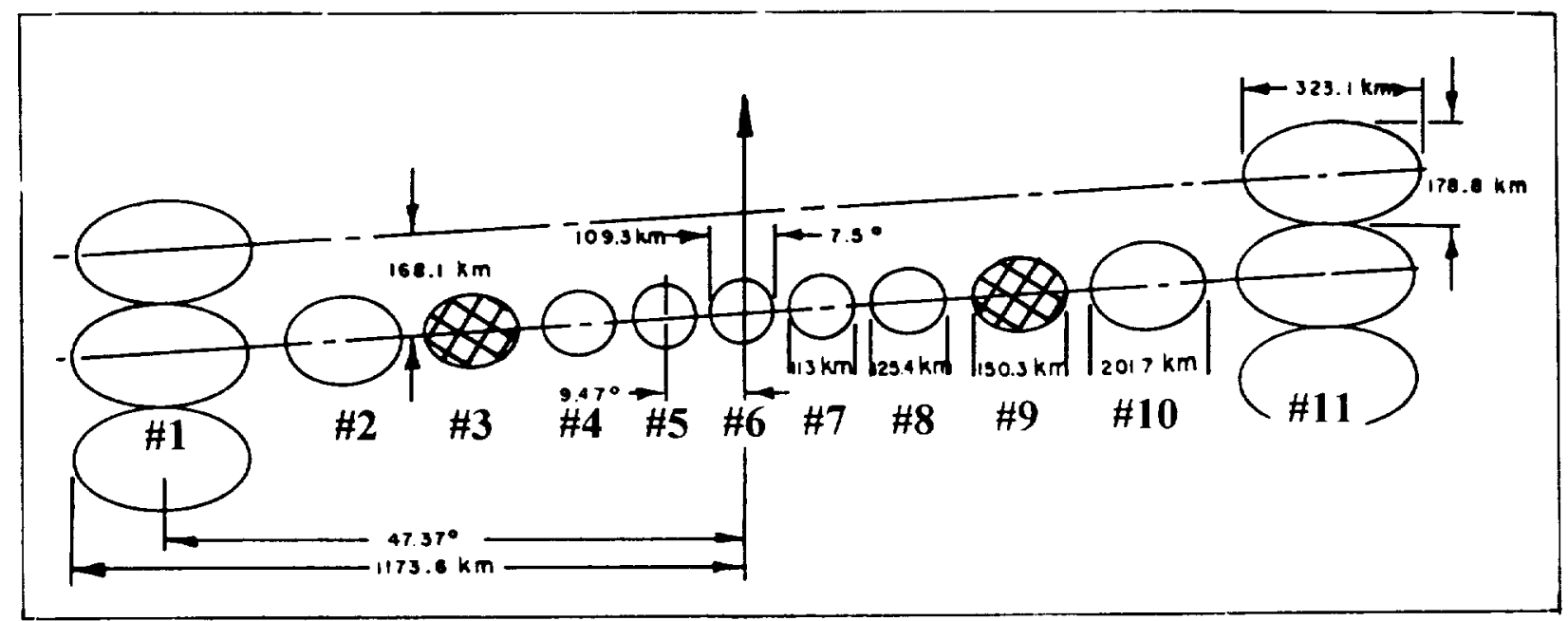

FIG. 2. MSU scan geometry and footprint half-power beamwidth spatial resolutions at the earth's surface (adapted from Grody 1983). View angle corrections in this study were performed to match all other beam positions to positions 3 and 9 .

footprint spacing is approximately $150 \mathrm{~km}$ in both along-track and cross-track directions. The MSU brightness temperatures $T_{b}$ were calibrated according to the procedure outlined in Spencer et al. (1990), although here an average of three scan lines of calibration information, instead of only one, were used to calibrate each scan line of data. This removes small scan-to-scan biases that are the result of insufficient radiometer dwell time when viewing the warm calibration load and the cosmic background. Unlike the long-term temperature monitoring of Spencer and Christy (1992a,b), the geostrophic vorticity, thermal wind, and vertical motion fields described here are all calculated from spatial gradients in the data. This makes absolute offsets in calibration between instruments nearly irrelevant to the computation of these fields for either weather or climate studies. Thus, the procedures described below could even be used for climate monitoring of these parameters without intercalibration between successive satellite sensors.

All satellite temperature sounders flown to date have been through-nadir scanners, resulting in a dependence of the weighting function height on view angle, which is due to the greater atmospheric path length as the instrument scans off-nadir. Thus, spatial variability in the satellite temperatures across the data swath is usually dominated by "limb darkening' resulting from the higher, off-nadir weighting functions sampling cooler air. In order to discuss the MSU data in pressure coordinate system terms, this effect must be corrected for by adjusting all the different view angles to correspond to the same pressure altitude. Indeed, the multiplicity of spatial gradient operators inherent in the computation of vertical velocity from (1) makes the limb correction procedure critical. Therefore, as long as through-nadir scanners produce the data, limb corrections will probably remain a necessary part of the data analysis procedures in order to allow discussion of baroclinic structures in a pressure coordinate system.

MSU channels 2, 3, and 4 (MSU2, MSU3, and MSU4) are used together to provide view angle corrections for MSU2 and MSU3. Rather than the traditional approach of correcting the nonnadir measurements to nadir, we correct to the intermediate earth incidence angle of $33^{\circ}$ ( footprint positions 3 and 9 in Fig. 2 ). This provides somewhat better performance than a correction to the nadir view since it involves a smaller range of deviations from the view angle to which it is corrected. Separate sets of limb correction coefficients $a$ are derived with multiple linear regression for each $4^{\circ}$ latitude band, month, satellite direction (ascending or descending paths), and separate satellite to correct $T_{b}$ to the $33^{\circ}$ incidence angle. This is done by regressing the average of print positions 3 and $9 T_{b}$ (each having a $33^{\circ}$ earth incidence angle) against the other incidence angles' average $T_{b}$,

$$
T_{b}^{*}=a_{0}+a_{2} T_{b 2}+a_{3} T_{b 3}+a_{4} T_{b 4},
$$

where $T_{b}^{*}$ is the view-angle-corrected $T_{b}$ for either MSU2 or MSU3. (The asterisk will be dropped from here onward, and all references to $T_{b}$ will assume limb corrections have been made.) The ability of (2) to provide useful view-angle corrections is a result of two effects: the averaging kernel effect of combining overlapping weighting functions into a new weighting profile, and the interlayer correlations of temperature providing information on one layer from another.

The lower-troposphere deep-layer temperatures we will show are the result of a linear combination of MSU2 and MSU3 (Fig. 1), which can be deconvolved into an averaging kernel (channel " $2 / 3$ ", $T_{b 2 / 3}$ $=1.6 T_{b 2}-0.6 T_{b 3}$ ) that has most of its sensitivity be- 
low $400 \mathrm{hPa}$. The upper-tropospheric/lower-stratospheric deep-layer temperatures are estimated directly from view-angle-corrected MSU3 data.

\section{c. MSU oceanic rainfall}

MSU-diagnosed oceanic rainfall fields were key to our awareness of the information content of deep-layer temperatures concerning quasigeostrophic vertical motions. This cloud water and rainwater emission signature is obtained primarily from MSU1. At $50.3 \mathrm{GHz}$ over the ocean, variability in MSUl $T_{b}$ comes from rainwater, cloud water, water vapor, and ocean surface roughness (wind). In these respects, MSU1 is similar to the $37-\mathrm{GHz}$ channel of the Special Sensor Microwave/Imager (SSM/I), except for some additional sensitivity to temperature due to its proximity to the 60-GHz oxygen-absorption complex.

While there is not enough spectral information to separate these various signatures with the MSU as there is with the SSM/I, the warmest MSU1 $T_{b}$ provide a useful index of rainfall activity. This was demonstrated by Spencer (1993) who quantified the warming signal with 122 maritime gauges compared with $7-10$ years of MSU data. The precipitation amount is calculated as a linear function of the excess $T_{b 1}$ warming above a $15 \%$ cumulative frequency distribution threshold after correction for the airmass temperature $T_{b 2 / 3}$ (see Spencer 1993 for further details ).

It is important to note that the quasigeostrophic concepts discussed below do not depend upon the accuracy of the MSU rainfall algorithm or its sensitivity to nonrain effects. It is included to demonstrate the inductive connection between an index of upward motion (the rainfall estimates ) and spatial patterns in the MSU temperature data. As an example of the algorithm's sensitivity to nonrain effects, radiative transfer calculations ( see Wentz 1983) suggest that assuming an average surface wind speed of $5 \mathrm{~m} \mathrm{~s}^{-1}$, the wind would need to increase to nearly $20 \mathrm{~m} \mathrm{~s}^{-1}$ for $T_{b 1}$ to reach the $15 \%$ cumulative frequency distribution threshold, at which point a nonzero rain value would be assigned.

\section{d. Satellite data gridding and finite differencing}

The satellite measurements were binned into a $0.5^{\circ}$ latitude-longitude grid based upon the NESDIS-provided locations of the MSU footprint centers. Even though $1.0^{\circ}$ or $1.5^{\circ}$ grid resolution would more closely approximate the MSU footprint size and spacing (see Fig. 2), $0.5^{\circ}$ was chosen to allow accurate spatial partitioning of each footprint's energy and to thus avoid significant errors in the computed temperature gradients. Empty grid squares were zonally interpolated to from nonempty grids up to $2.5^{\circ}$ away in both east and west directions. To help smooth out random errors in the footprint data, the $T_{b}$ fields are then spatially smoothed with a $3.5^{\circ}$ latitude by $5.5^{\circ}$ longitude Gaus- sian-shaped filter that applies less weight for data at a farther distance from the center grid square location. Finite differences were taken at $500-\mathrm{km}$ spacing, independent of latitude, but were applied every $0.5^{\circ}$ in latitude and longitude. Note that the spatial resolution inherent in the MSU footprint size, spacing, and our smoothing filter makes the resulting $T_{b}$ fields quite smooth at $0.5^{\circ}$ grid resolution.

\section{Satellite image analysis}

While the results of this study might have been deduced from theoretical considerations alone, they were largely an inductive result of visual inspection of $6-\mathrm{h}$ imagery of the three different MSU fields. We examined numerous cases of cyclone development and their associated MSU temperature and rainfall patterns. A particularly strong North Pacific cyclone is shown in Fig. 3 at 12-h intervals during its development on 1315 March 1983. The lower-tropospheric temperatures ( $T_{b 2 / 3}$, dashed) reveal a thermal trough that sharpens considerably during cyclone intensification. The upper-troposphere/lower-stratosphere temperature ( $T_{b 3}$, solid) field reveals a lobe of warmth extending southward over the trough from a warm feature to the north. The $T_{b 3}$ warm lobe also intensifies during the period. The MSU-diagnosed rain rates ( $\mathrm{mm} \mathrm{h}^{-1}$, heavy dotted) indicate that the primary rain activity is located to the east of both the lower-tropospheric thermal trough and the upper-tropospheric/lower-stratospheric warm lobe. Through inspection of this and many other cases it was consistently observed that extratropical regions of $M S U$-estimated rainfall usually correspond to regions where the $T_{b 2 / 3}$-inferred midtropospheric flow (thermal wind) "blows' "across a $T_{b 3}$ gradient in a direction from warmer to cooler upper-tropospheric/ lower-stratospheric temperatures. Note the tendency for the rainfall to occur in a regions of positive $T_{b 3} / T_{b 2 / 3}$ solenoids. Examples of similar relationships were present on every day for which global imagery was examined. While the case illustrated in Fig. 3 is comparatively intense, many examples were found where the amplitudes of the features were much smaller, yet coherence in the spatial relationships among the thermal structures and the rainfall fields remained the same.

While it is well known that the lower-tropospheric deep-layer temperatures such as $T_{b 213}$ provide a useful estimate of lower-tropospheric thickness, it has been less apparent what the significance is of the upper-tropospheric temperature patterns observed by MSU3. Conventional wisdom has maintained that MSU3 data are difficult to interpret because the channel 3 weighting function straddles the midlatitude tropopause. However, its potential importance in the formation of intense extratropical cyclones was addressed by Velden (1992), who showed that MSU3 warm pools revealed by this channel were associated with cyclone development at the surface, with the warm pools upwind of the cyclone during the 
a. 1919 UTC 13 March

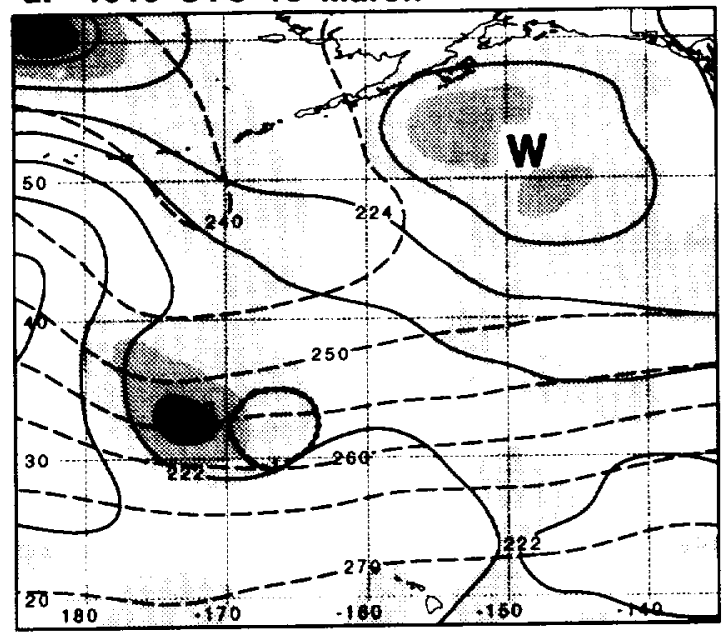

c. 1855 UTC 14 March

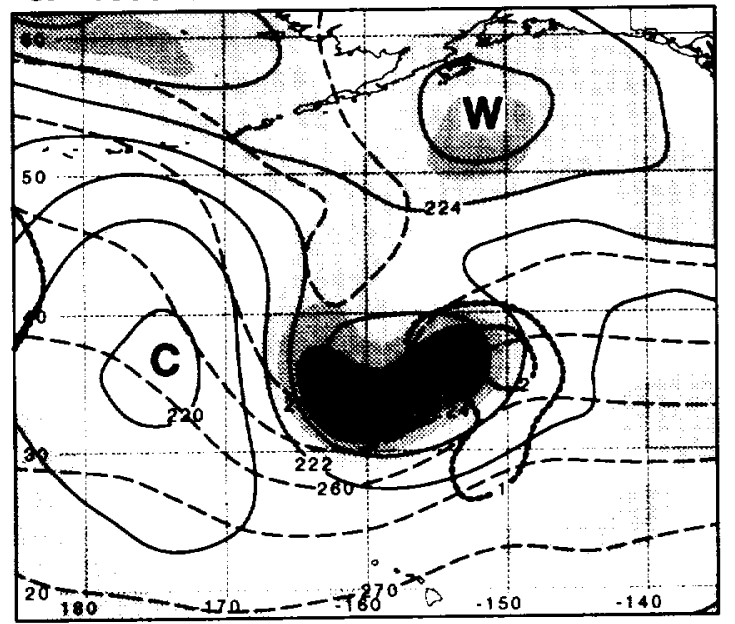

b. 0637 UTC 14 March

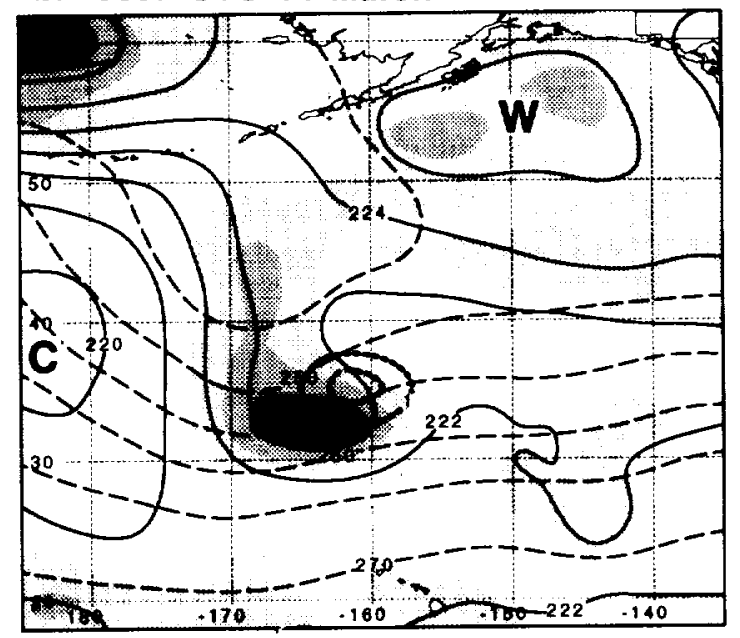

d. 0613 UTC 15 March

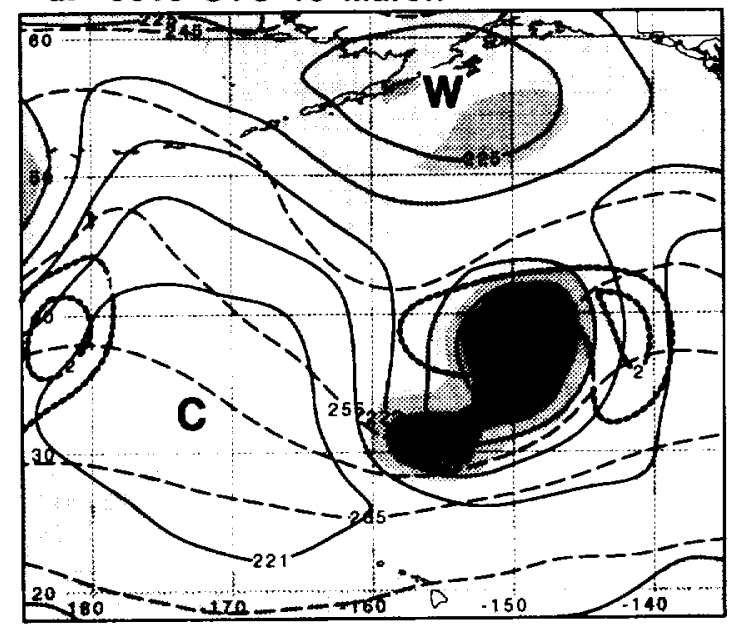

Fig. 3. MSU deep-layer temperatures of the lower troposphere $T_{b 273}$ (K, dashed); upper troposphere/lower stratosphere $T_{b 3}$ (K, solid); positive geostrophic relative vorticity (every $4 \times 10^{-5} \mathrm{~s}^{-1}$, shaded); and MSU1 rainfall patterns ( $\mathrm{mm} \mathrm{h}^{-1}$, shaded) observed approximately every $12 \mathrm{~h}$ during the development of a North Pacific cyclone (13-15 March 1983). The observation times listed are for the satellite passes nearest $30^{\circ} \mathrm{N}, 170^{\circ} \mathrm{W}$.

intensification phase, then moving over the cyclone by the mature phase. While Velden suggested that these warm pools were associated with tropopause undulations, that is, regions of depressed tropopause and adiabatically warmed upper-tropospheric and lower-stratospheric air, we believe a more general and quantitative explanation of these features is possible.

Note that there is a similarity between these MSU relationships seen in Fig. 3 and the omega forcing term in Eq. (1) (the advection of geostrophic vorticity by the thermal wind). In this context, if the MSU rainfall (vertical motion) is located where the $T_{b 2 / 3}$ isotherms (thermal winds) cross the $T_{b 3}$ isotherms, could the $T_{b 3}$ data then contain information on midtropospheric vorticity? We believe that the supposed difficulty in interpretation posed by the MSU3 weighting function strad- dling the tropopause is actually a red herring. The construction of a geopotential height field (which defines the three-dimensional structure of a baroclinic system) from radiosonde data, does not depend upon identifying the tropopause. Similarly, a microwave sounder weighting function, to the extent that it provides a deeplayer average temperature pattern (approximating a thickness pattern), also provides a geopotential height pattern (assuming a sufficient boundary condition), from which the geostrophic vorticity can be computed.

\section{Midtropospheric vorticity patterns from the MSU \\ a. Computation from MSU3}

A traditional approach to diagnosing midtropospheric heights (or the corresponding geostrophic vor- 


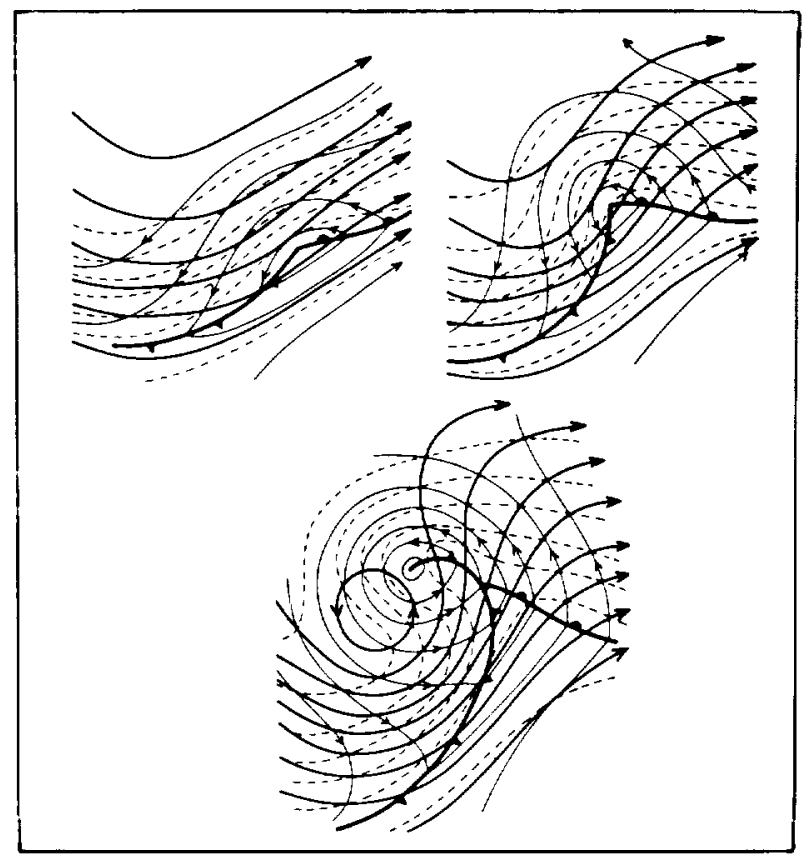

FIG. 4. Palmen and Newton's (1969) depiction of the relationships between 500-hPa heights (heavy solid lines); $1000-500-\mathrm{hPa}$ thickness (dashed lines); and sea level isobars (light solid lines) at three stages of cyclone development.

ticity) from satellite-derived thermal or thickness fields would proceed along the following familiar lines: the $500-\mathrm{hPa}$ height field can be built hydrostatically by adding the $1000-500-\mathrm{hPa}$ thickness field (approximated by $\left.T_{b 2 / 3}\right)$ to the 1000 -hPa height field, for example, the surface pressure patterns in Fig. 4. In this approach, solving the hypsometric equation, which is a boundary value problem, starts from the bottom up, and we would need knowledge of the surface pressure field as a lower boundary condition. Unfortunately, there is currently no method for measuring surface pressure patterns from satellite data.

However, we noticed that during the development of the waves, the upper-tropospheric warm features were centered somewhat downstream (east) of the trough in the lower-tropospheric channel $2 / 3$ thermal wave, as is most obvious in Figs. $3 \mathrm{~b}$ and $3 \mathrm{c}$. In a developing baroclinic disturbance, this is precisely where thermal wind balance tells us that a midtropospheric height minimum should be found. The classic example (Fig. 4) shown by Palmen and Newton (1969) illustrates the phase lag between the $500-\mathrm{hPa}$ height field and $1000-500-\mathrm{hPa}$ thickness field and its relationship to the surface low. Since surface pressure decreases to the east of the thickness trough or, equivalently, thicknesses decrease to the west of the surface low, the midtropospheric height minimum must lie somewhere in between the surface low and the upstream thickness trough. But if MSU3 provides an estimate of upper-tropospheric thickness, why are warm anomalies in this field consistently located where the bottom-up approach tells us where the height field minimum (or vorticity maximum) should be?

To examine this question, we appeal to Hirshberg and Fritsch's (1991a,b; 1993) findings with regard to diagnosing midtropospheric height features from temperature changes above a pressure level. Whereas the traditional approach assumes a lower-boundary condition of height invariance, HF91 and HF93 discussed the alternative of an upper-boundary condition where the amplitude of synoptic wave structure effectively vanishes - the level of insignificant dynamics (LID). If a LID exists, say near $50 \mathrm{hPa}$, where cyclone-scale height variations are relatively small, then the $400-\mathrm{hPa}$ height field features can be estimated from the average temperature between $400 \mathrm{hPa}$ and the LID, that is, the thickness, which is closer to what microwave sounding channels actually measure. H93 discusses the fact that the LID hypothesis is only valid for spatial scales smaller than about $4000 \mathrm{~km}$. Indeed, we found from cases like that shown in Fig. 3 that only the cyclonescale (not planetary-scale) features in $T_{b 3}$ had any apparent relationship with the MSU rainfall patterns. That is, rainfall was more likely to be present when the $T_{b 2 / 3}$-inferred thermal wind was exiting a cyclone-scale (not planetary scale) warm $T_{b 3}$ feature. We therefore hypothesize that because cyclone-scale height features are weak near the top of the channel 3 weighting function, cyclone-scale patterns in $T_{b 3}$ can be interpreted as cyclone-scale height patterns near the bottom of the channel 3 weighting function (near $400 \mathrm{hPa}$ ).

This is illustrated in Fig. 5, a conceptual diagram of height features in the 50 - and 400 -hPa surfaces. If we can speak in terms of two size categories of waves, planetary-scale and cyclone-scale, then both the 50and $400-\mathrm{hPa}$ surfaces will in general have planetary wave structures (which are not necessarily the same),

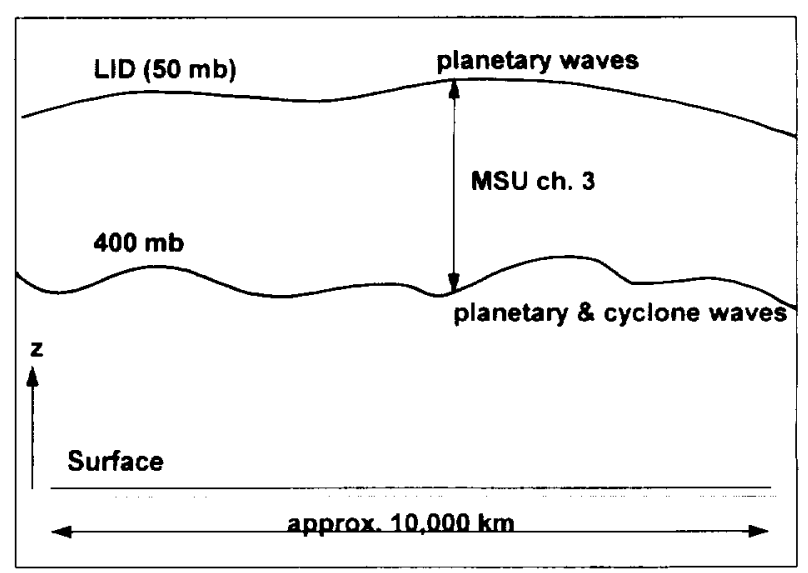

FIG. 5. Conceptual diagram of wave (height) structures in the 400 and 50 -hPa surfaces; the layer is measured approximately by MSU channel 3. 
but only the $400-\mathrm{hPa}$ surface will exhibit cyclone-scale features. Planetary waves assume prominent amplitudes throughout the stratosphere, so we cannot determine all spatial scales of the midtropospheric height field from MSU3, that is, we cannot interpret the solid lines in Fig. 3 as geopotential height contours. But because the geostrophic vorticity of large, planetary-scale features will be weak, quantitative information of the cyclone-scale features can be extracted if the geostrophic vorticity is computed. We would expect that the Laplacian operator used for computation of geostrophic vorticity on the rhs of ( 1 ) would be dominated by cyclone-scale features with little contribution from planetary wave features. If the LID assumption is valid, then the height field at the bottom of the layer sensed by MSU3 will have the same small-scale horizontal structure as the "thickness" field represented by the MSU3 weighting function. Then the vorticity term in (1) can be approximated by replacing the geopotential height by satellite-sensed deep-layer temperature,

$$
\zeta_{s}=\frac{1}{f} \nabla^{2} \Phi \approx \frac{1}{f} \nabla^{2}\left[T_{b 3}\left(R \ln \frac{p_{2}}{p_{1}}\right)\right],
$$

where $p_{2}=50 \mathrm{hPa}, p_{1}=400 \mathrm{hPa}$. As discussed above, the bounding pressure level values for $p_{2}$ and $p_{1}$ would be strictly accurate only if the satellite could actually measure the 400-50-hPa thickness. Because the MSU3 weighting function does not have a "boxcar" shape, these reference levels are not strictly applicable and are just approximations at this point. The Laplacian operator was computed with five-point finite differencing at fixed (latitude-independent) $500-\mathrm{km}$ gridpoint spacing but applied at every $0.5^{\circ}$ grid point. The large space scale for this Laplacian operator was necessary to suppress small-scale noise, and the resulting vorticity field will represent only those features larger than about $1000 \mathrm{~km}$ in size.

The shaded regions in Fig. 3 indicate positive geostrophic relative vorticity thus computed from MSU3. It can be seen that the vorticity influence of the largerscale patterns in $T_{b 3}$ is weak, while the cyclone-scale vorticity maxima resulting from smaller-scale perturbations in the $T_{b 3}$ field are brought out by the Laplacian operator. A good example of the interpretational superiority of the vorticity field over the raw MSU3 field is the secondary vorticity maximum that plunges southeastward near $170^{\circ} \mathrm{W}$ ( Fig. 3) toward the primary vorticity maximum, intensifying and rotating through the trough during the period.

Now the advection of vorticity by the thermal wind can be visualized by following the $T_{b 2 / 3}$ contours (dashed) from higher (darker shading) vorticity values to lower (lighter shading) vorticity values. The rainfall areas are seen to occupy areas where there is positive vorticity advection by the thermal wind. The most prominent exception is the lobe of rainfall in Fig. 3c, which extends southwestward from the main vorticity advection region. This is probably a line of deep convection along the cold front, possibly where the (neglected) deformation terms in (1) are large.

\section{b. Comparisons during ERICA}

The MSU-computed midtropospheric geostrophic vorticity was compared to that computed from radiosonde data with three cases from ERICA (Experiment on Rapidly Intensifying Cyclones over the Atlantic) (Hadlock and Kreitzberg 1988). These include two cases from 1988: 1200 UTC 13 December (Fig. 6) and 1200 UTC 17 December (Fig. 7); and 0000 UTC 4 January 1989 (Fig. 8). Geostrophic vorticity fields were computed from ( 3 ) using the MSU data recorded near these analysis times. Geostrophic vorticities were also computed from the NMC Global Data Assimilation System (GDAS) analyses, with layer temperatures weighted by the MSU3 weighting function shown in Fig. 1. While the GDAS also utilizes tropospheric MSU data (but over the oceans), the weight it is given is so small that the GDAS and MSU fields can be considered nearly independent (NOAA/NWS/NMC 1994, personal communication ). Also computed were the lowertropospheric temperature patterns for the channel $2 / 3$ weighting profile shown in Fig. 1.

All three cases (Figs. 6-8) involved a vorticity maximum embedded in westerly to northwesterly flow. The MSU features are all displaced to the east of the NMC features due to the satellite data being one to three hours later than the analysis times. The MSU and NMC vorticity patterns are basically similar, with some small differences in magnitude and shape of the vorticity features. These differences are important, though, in the context of diagnosing quasigeostrophic vertical motions from (1), which can be estimated by the advection patterns produced by the vorticity and thermal wind implied by the selenoids on the left-hand sides of Figs. 6-8. Many other visual comparisons to operationally available daily Nested Grid Model (NGM) analyses over North America (not shown) almost always revealed excellent agreement with the NGM 500$\mathrm{hPa}$ vorticity analysis.

\section{Vertical motion calculations}

To diagnose $\omega$, we rewrite (1) as

$$
\begin{aligned}
\omega \approx-\left\{\sigma\left[\frac{8}{(1000 \mathrm{~km})^{2}}\right]\right. & \left.+f^{2}\left[\frac{64}{(1000 \mathrm{hPa})^{2}}\right]\right\}^{-1} \\
\times & 2 f \frac{\Delta \mathbf{V}_{g}}{\Delta p} \cdot \nabla\left(\frac{1}{f} \nabla^{2} \Phi\right)
\end{aligned}
$$

where $f$ is used instead of $f_{0}$ to allow computations over all extratropical latitudes. The three-dimensional Laplacian operator in (1) is now used to scale the calculations of $\omega$, assuming a single sinusoidal spatial 


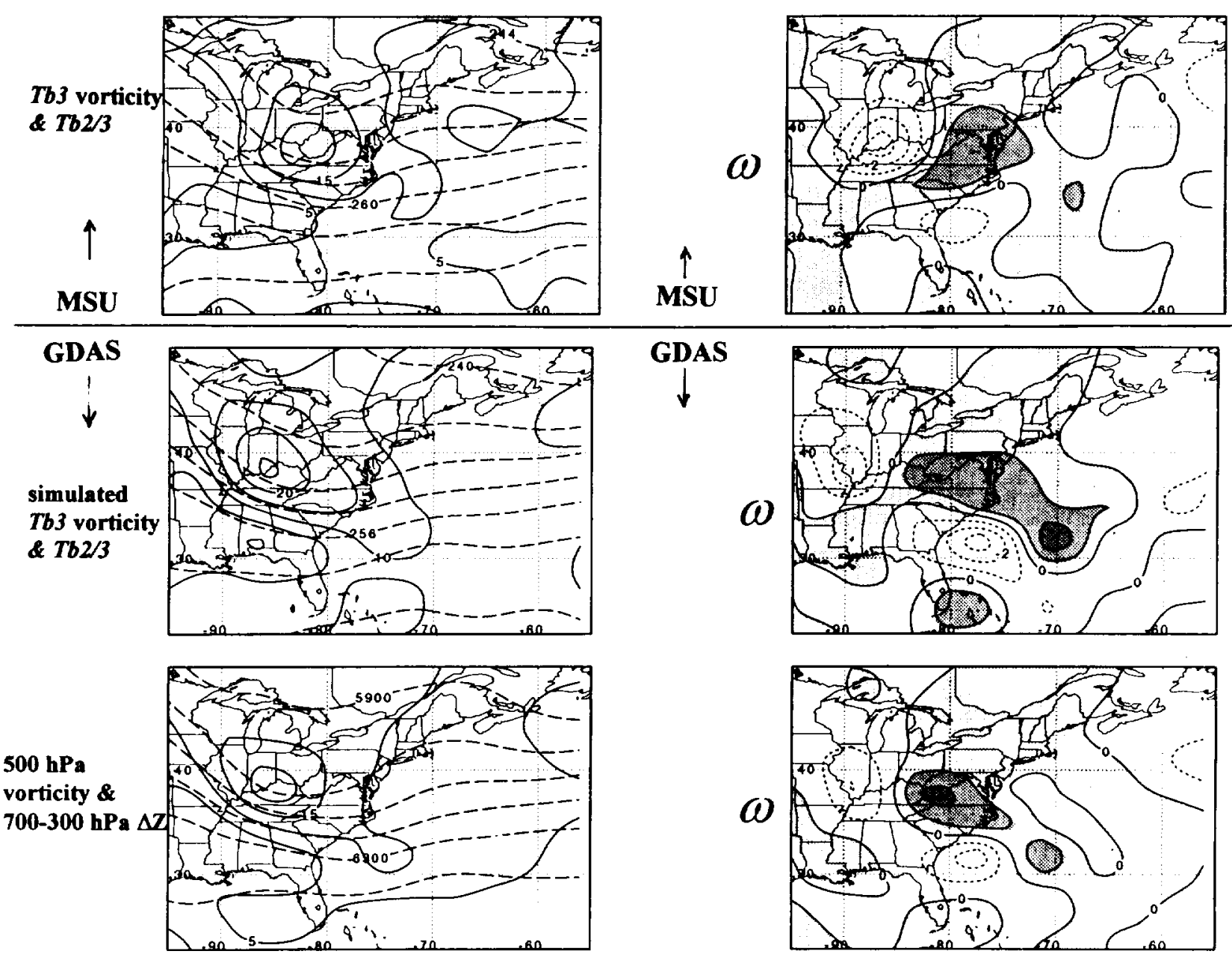

FIG. 6. MSU and NMC GDAS geostrophic vorticity, thermal field, and vertical motions (omega) for 1200 UTC 13 December 1988. Top two panels: MSU-derived lower-tropospheric temperatures $T_{b 23}$ and geostrophic vorticity computed from $T_{b, 3}$ and the corresponding omega field $\left(\mu \mathrm{b} \mathrm{s}{ }^{-1}\right)$. Middle two panels: the corresponding fields computed from the NMC GDAS analysis after applying the weights in Fig. 1 to analysis field mandatory level temperatures and the corresponding omega field. Bottom two panels: 500-hPa geostrophic vorticity and 700$300-\mathrm{hPa}$ thickness field and the corresponding omega field. All omegas are computed with Trenberth's (1979) approximation of the omega equation.

mode, with the vertical profile of omega peaking near $500 \mathrm{hPa}$ and going to zero at 1000 and $0 \mathrm{hPa}$ and assuming a horizontal wavelength of $1000 \mathrm{~km}$. The corresponding numerical constants are addressed by Bluestein $(1992,332-333)$. The static stability parameter in $(4)$ is allowed to vary with airmass temperature:

$$
\sigma \approx\left(\frac{100 \mathrm{~m}^{2}}{s^{2} \mathrm{hPa}}\right)\left(\frac{1}{\theta} \frac{\partial \theta}{\partial p}\right)
$$

where

$$
\theta \approx T_{b 2 / 3}+50^{\circ} \mathrm{C}
$$

and

$$
\frac{\partial \theta}{\partial p}=0.066+0.001\left(275-T_{b 2 / 3}\right)
$$

are computed from the typical range of tropical to polar $T_{b 2 / 3}$ and their corresponding potential temperature characteristics are estimated from a thermodynamic diagram. The thermal wind term in (4) is computed from the MSU lower-tropospheric deep-layer measurements:

$$
\frac{\Delta \mathbf{V}_{g}}{\Delta p}=\frac{1}{p_{0}-p_{1}} \frac{1}{f} \mathbf{k} \times \nabla\left(R \ln \frac{p_{1}}{p_{0}} T_{b 2 / 3}\right),
$$

where $p_{0}=1000 \mathrm{hPa}$ and $p_{1}=400 \mathrm{hPa}$.

The vertical motion fields diagnosed from the solenoids of geostrophic vorticity and $T_{b 2 / 3}$ temperature patterns on the left-hand side of Figs. 6, 7, and 8 are shown on the right-hand sides of the same figures. As was the case for vorticity, there is basic agreement between the MSU and NMC patterns of rising and sinking motion for all three days. The MSU again shows an 

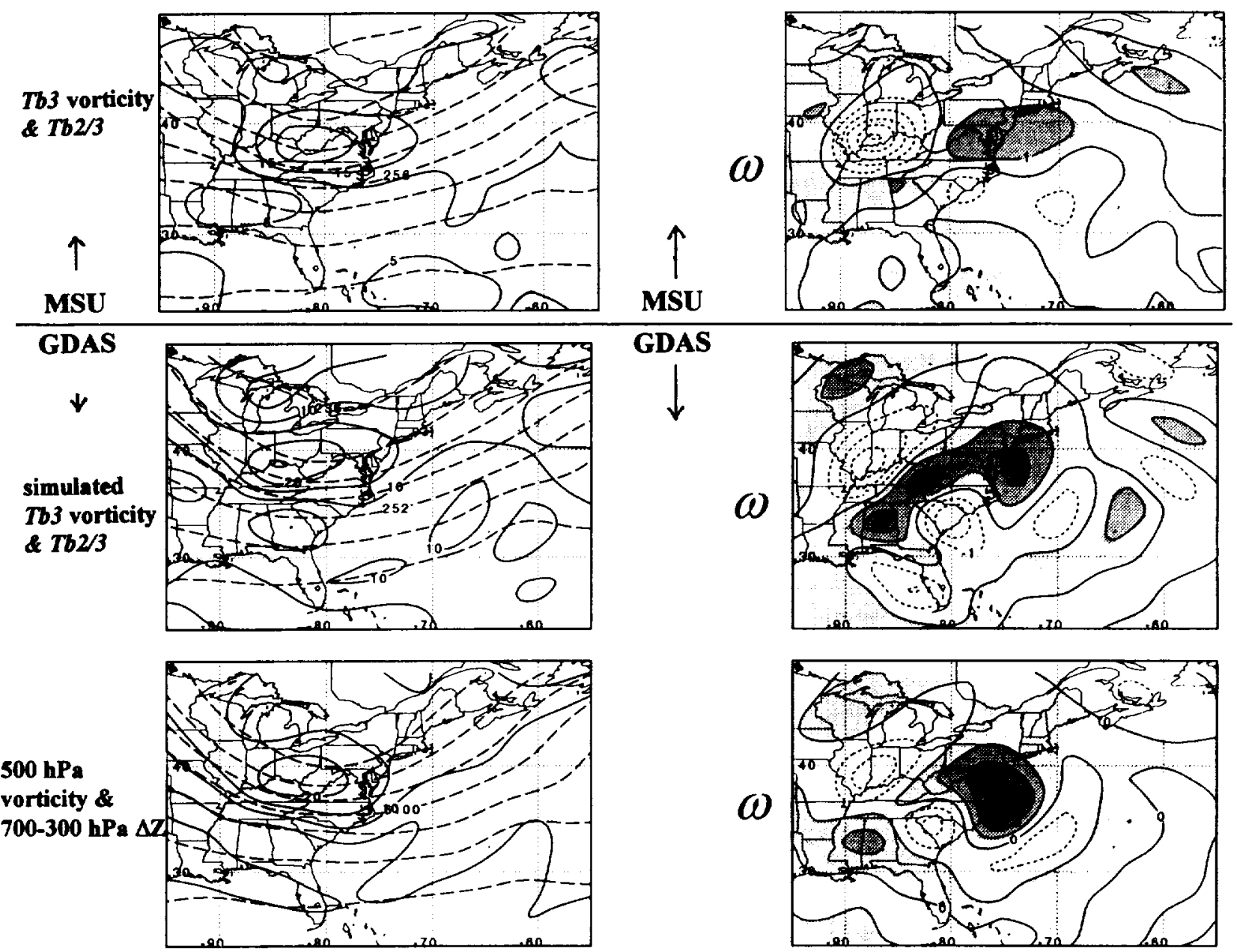

FIG. 7. As in Fig. 6 except for 1200 UTC 17 December 1988.

eastward offset of its features, which is related to its data being collected $1-3 \mathrm{~h}$ later than the analysis times of 0000 and 1200 UTC.

The largest source of error in the computation of omega from the MSU data comes from the geostrophic vorticity field and thus from limb correction errors in $T_{b 3}$. This is because the geostrophic vorticity gradient is directly proportional to a third spatial derivative of the $T_{b 3}$ field, whereas the thermal wind calculation from the lower-tropospheric temperatures involves only a first spatial derivative of $T_{b 2 / 3}$. Through examination of many cases (not shown), the majority of significant vorticity errors were confined to the swath edges, in particular the extreme (numbers 1 and 11 ) MSU footprint positions. Very careful limb-correction procedures can ensure that these errors are relatively random but cannot eliminate them. Nevertheless, we retained the extreme footprint position data in our study to examine how well the technique would perform with the full set of MSU data, which provides essentially complete coverage of the extratropics. Better limb correc- tions will require more radiometer channels with overlapping weighting functions, lower instrumental noise, or the flight of conically scanning temperature sounders that would eliminate the need for limb corrections (though at the expense of a somewhat narrower swath width).

\section{Summary and conclusions}

We have explored the information content of satellite-measured deep-layer temperatures in the context of quasigeostrophic dynamical concepts. Midtropospheric geostrophic vorticity can be estimated from a single temperature sounder channel (MSU3). The conventional wisdom that "it is difficult to interpret MSU3 because it straddles the tropopause' 'is irrelevant to discussion of geopotential height patterns, which are computed from a vertical integration of temperature (which a microwave temperature sounder approximates) in combination with a suitable upper boundary condition. The thermal structure that MSU3 responds to in pres- 

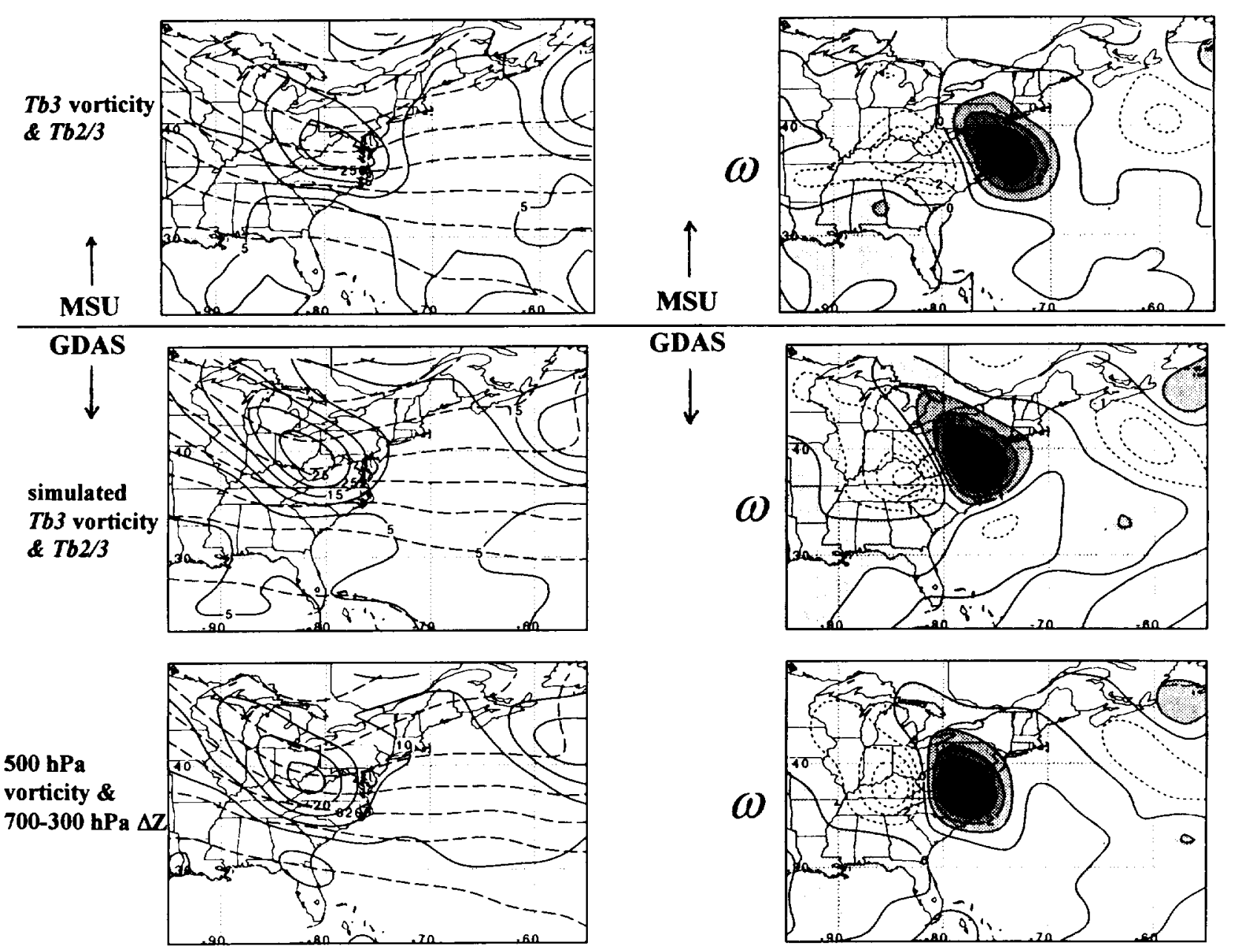

FIG. 8. As in Fig. 6 except for 0000 UTC 4 January 1989.

sure coordinates has cyclone-scale variability only near the bottom of the layer, while the top corresponds to the stratospheric LID where height variations on the cyclone scale are minimal. Because the Laplacian operator used in geostrophic vorticity calculations has inherent scale selectivity, geostrophic vorticities calculated from the MSU3 temperature field are relatively insensitive to the planetary-scale features and extract the cyclone-scale features. Indeed, the good agreement between radiosonde-based and satellite-based calculated patterns of geostrophic vorticity are a measure of the validity of the LID assumption.

When this midtropospheric geostrophic vorticity is combined with thermal wind estimates from lower-tropospheric deep-layer temperatures, we have demonstrated that it is possible to diagnose quasigeostrophic vertical motions. While the vertical motion technique might have been anticipated on theoretical grounds alone, it was visual examination of the MSU1 rainfall fields that implied a strong connection between tropospheric ascent and the midtropospheric thermal wind blowing from warm to cool upper-tropospheric/lowerstratospheric temperature. Therefore, it is significant the MSU data themselves revealed a relationship between vertical motion and geostrophic vorticity advection by the thermal wind. That this quasigeostrophic relationship is a major feature of MSU-observed extratropical deep-layer temperature patterns reaffirms the Trenberth (1978) simplification of the quasigeostrophic omega equation as a powerful conceptual tool.

The largest source of error in the calculations of vertical motion originates from the geostrophic vorticity field computed from MSU3. Because this portion of the omega equation involves the horizontal gradient of a Laplacian (i.e., three gradient operators) of the MSU3 $T_{b}$ field, small errors in the satellite data are magnified. In particular, the limb-correction procedure for MSU3 is critical, and single-footprint radiometric noise must be at least as good as that of the MSU $\left(0.2^{\circ}\right)$. Some improvement in geostrophic vorticity calculations can be expected when the Defense Meteoro- 
logical Satellite Program's Special Sensor Microwave/ Imager Sounder (SSM/IS) flies, due to its conical scan pattern, thus eliminating the need for limb corrections. Also, the Advanced Microwave Sounding Unit (AMSU), scheduled to replace the MSU in 1996, will have more channels with overlapping weighting functions, thus allowing better limb corrections than is possible with the MSU.

The ability to measure dynamical quantities such as geostrophic vorticity and vertical motion could allow satellite-only surveys of baroclinic transients and their regional organization into storm tracks since the beginning of global satellite coverage in 1979, including their seasonal and interannual variability. These data would be an important complement to assimilated fields that contain the uncertainties of physics formulation and climate bias in the assimilation model, arbitrary values in analysis quality weights, and evolving makeup of the input data stream. If geostrophic vorticity and vertical motion fields can be produced from satellite data alone, the resulting source of independent information could help verify and possibly improve the physics and parameterizations in climate and weather prediction models.

It is not yet known whether there are special synoptic conditions wherein the above procedures do not provide a good estimate of midtropospheric geostrophic vorticity and vertical motion. One possible scenario would be a very shallow baroclinic disturbance that is not well resolved by the satellite deep-layer temperatures and that might have a vertical motion maximum well below the midtroposphere. Neither have we examined how much of the error in these methods is due to the weighting function (or averaging kernel) shapes not providing an accurate measure of thickness. More studies would be required to examine these questions.

Acknowledgments. We thank $\mathrm{F}$. Wentz for radiative transfer calculations of the effect of ocean surface roughness on MSU1. This work has benefited from years of advice and guidance provided to the first author by N. Grody. This work was supported by the Office of Mission to Planet Earth at NASA Headquarters.

\section{REFERENCES}

Bluestein, H. B., 1992: Synoptic-Dynamic Meteorology in Midlatitudes. Oxford University Press, $431 \mathrm{pp}$.
Conrath, B. J., 1972: Vertical resolution of temperature profiles obtained from remote radiation measurements. J. Atmos. Sci., 29, $1262-1271$

Durran, D. R., and L. W. Snellman, 1987: The diagnosis of synopticscale vertical motion in an operational environment. Wea. Forecasting, 2, 17-31.

Eyre, J. R., and A. C. Lorenc, 1989: Direct use of satellite sounding radiances in numerical weather prediction. Meteor. Mag. 118, $13-16$.

Grody, N. C., 1983: Severe storm observations with the Microwave Sounding Unit. J. Climate Appl. Meteor., 22, 609625.

Hadlock, R., and C. W. Kreitzberg, 1988: The Experiment on Rapidly Intensifying Cyclones over the Atlantic (ERICA) field study: Objectives and plans. Bull. Amer. Meteor. Soc. 69, $1309-1320$.

Hirshberg, P. A., and J. M. Fritsch, 1991 a: Tropopause undulations and the development of extratropical cyclones. Part I: Overview and observations from a cyclone event. Mon. Wea. Rev., 119, 496-517.

- and $-1991 \mathrm{~b}$ : Tropopause undulations and the development of extratropical cyclones. Part II: Diagnostic analysis and conceptual model. Mon. Wea. Rev., 119, 518-550.

$\longrightarrow$, and -1993 : On understanding height tendency. Mon. Wea. Rev. 121, 2646-2661.

Holton. H. R., 1979: An Introduction to Dynamic Meteorology. Academic Press, $391 \mathrm{pp}$.

Hoskins, B. J., I. Draghici, and H. C. Davies, 1978: A new look at the $w$-equation. Quart. J. Roy. Meteor. Soc., 104, $31-38$.

Huang. H.-L., W. L. Smith, and H. M. Woolf, 1992: Vertical resolution and accuracy of atmospheric infrared sounding spectrometers. J. Appl. Meteor., 31, 265-274.

Palmen, E., and C. W. Newton, 1969: Atmospheric Circulation Systems. Academic Press, 603 pp.

Spencer, R. W., 1993: Global oceanic precipitation from the MSU during 1979-91 and comparisons to other climatologies. J. Climate, $7,1301-1325$.

, and J. R. Christy, 1992a: Precision and radiosonde validation of satellite gridpoint temperature anomalies. Part I: MSU channel 2. J. Climate, 5, 847-857.

$\longrightarrow$, and — 1992b: Precision and radiosonde validation of satellite gridpoint temperature anomalies. Part II: A tropospheric retrieval and trends $1979-90$. J. Climate, 5, 858866.

and N. C. Grody, 1990: Global atmospheric temperature monitoring with satellite microwave measurements: Method and results, 1979-84. J. Climate, 3, 1111-1128.

Sutcliffe, R. C., 1947: A contribution to the problem of development. Quart. J. Roy. Meteor. Soc., 73, 370-383.

Trenberth, K. E., 1978: On the interpretation of the quasigeostrophic omega equation. Mon. Wea. Rev., 106, 13I-137.

Velden, C. S., 1992: Satellite-based microwave observations of tropopause-level thermal anomalies: Qualitative applications in extratropical cyclone events. Wea. Forecasting, 7, 669682.

Wentz, F. J., 1983: A model function for ocean microwave brightness. J. Geophys. Res.. 88(C3), 1892-1908. 